\title{
Velopharyngeal Closure and Resonance in Children Following Early Cleft Palate Repair: Outcome Measurement
}

\author{
V. S. Aparna ${ }^{1}$ M. Pushpavathi ${ }^{2} \quad$ Krishnamurty Bonanthaya ${ }^{3}$ \\ ${ }^{1}$ Consultant Speech Language pathologist, Jain Unit of Smiletrain, \\ Bhagwaan Mahaveer Jain hospital, Bangalore, Karnataka, India \\ ${ }^{2}$ All India Institute of Speech and Hearing, Manasagangothri, \\ Mysuru, Karnataka, India \\ ${ }^{3}$ Consultant Maxillofacial Surgeon, Jain Unit of Smile Train, \\ Bhagwaan Mahaveer Jain Hospital, Bangalore, Karnataka, India
}

\begin{abstract}
Address for correspondence V.S. Aparna, MSc Speech Language Pathology, Consultant Speech Language Pathologist, Jain Unit of Smiletrain, Bhagwaan Mahaveer Jain Hospital, Bangalore 560052, Karnataka, India (e-mail: aparnaslp@bmjh.org).
\end{abstract}

\begin{abstract}
Keywords

- videofluoroscopy

- perceptual speech

- hypernasality

- nasal air emission

- closure ratio

Introduction Timing of cleft palate repair and the method of speech outcome measurement in children with cleft lip and palate are much debated topics. The associated problems and quality of life in these children depend on the timing of the surgery.

Aim The aim of this study was to investigate the velopharyngeal (VP) function and resonance parameters in children following early cleft palate repair.

Method A total of 25 Kannada-speaking children with early repaired cleft palate were subjected to speech assessment and videofluoroscopic assessment. Perceptual speech parameters measured were severity of hypernasality and presence of nasal air emission. Videofluoroscopy was interpreted in terms of closure ratios to predict the severity of VP dysfunction.

Results The analysis of videofluoroscopic images indicated that $48 \%$ of children had complete VP closure and 52\% had perceptually normal resonance. A good correlation was found between the closure ratio and hypernasality.

Conclusion Understanding the perceptual speech parameters and their structural correlates for outcome measurement will give better evidence for refining the existing treatment protocols. Data on a larger population are warranted for establishing predictors of optimum speech outcome.
\end{abstract}

\section{Introduction}

Speech problems in children with cleft lip and palate (CLP) are reported even after early primary cleft palate repair. Speech characteristics commonly observed in school-going children with repaired CLP (RCLP) are hypernasality, nasal air emission, and compensatory and obligatory articulatory errors. The most common resonance disorder reported due to velopharyngeal (VP) dysfunction (VPD) is hypernasality. This can be caused due to structural inadequacy or functional incompetence. ${ }^{1}$ Speech disorders in children with CLP can be assessed by subjective and objective methods. Perceptual assessment is considered as the gold standard for the assessment of speech and is often validated against instrumental measures, ${ }^{2-6}$ hence various protocols have been proposed for assessment of speech.

Perceptual speech parameters have been documented using various protocols to avoid language bias and make comparison across groups as well as centers possible. One of the widely accepted protocols for reporting speech outcome in individuals with CLP was proposed by Henningsson et al. ${ }^{7}$ The scale included five universal parameters, viz. hypernasality, nasal air emission, hyponasality, voice disorder, and consonant production error. Two global parameters, speech understandability and speech acceptability, were also included. Only a few studies have used this standard protocol for the assessment of speech in CLP.
DOI https://doi.org/ $10.1055 / \mathrm{s}-0039-1696608$ ISSN 0970-0358.
License terms

()(1) $\Theta \circledast$ 
Hypernasality and nasal air emission are often reported. Shprintzen $^{8}$ reported that 10 to $20 \%$ of children who had undergone early primary palatoplasty had persisting hypernasality, while the study by Sell ${ }^{4}$ in their large-scale outcome measurement in cleft care in the United Kingdom found that $45 \%$ of the children had nasal air emission. Hubbard et $\mathrm{al}^{9} \mathrm{con}-$ ducted a study to assess the efficacy of sphincter pharyngoplasty. They assessed the VP function and resonance parameters in 18 primarily operated children with CLP and found an average hypernasality score of 1.94 when measured in a 3-point rating scale. Twelve children were reported to have nasal air emission. Some studies ${ }^{10,11}$ have emphasized the importance of using instrumental assessments as complementary to perceptual documentation of speech outcomes.

The most widely used instrumentation for clinical examination of VP function is videofluoroscopy (VFS) and videoendoscopy in real time, ${ }^{12}$ which allows the imaging of the VP mechanism at rest and in motion. This information is critical in decision making for further management including speech therapy and surgical correction. ${ }^{13}$ Standardized guidelines for reporting the findings of nasopharyngoscopy and VFS have also been proposed by various authors. ${ }^{14,15}$

It is essential to arrive at a correlation between perceptual and instrumental analyses. Studies ${ }^{16-18}$ have established a positive correlation between hypernasality, nasal air emission, and VP orifice area. Lipira et $\mathrm{al}^{11}$ in their study aimed at comparing nasoendoscopy (NE), VFS, and perceptual speech assessment in subjects with VPD. Using instrumental assessment, outcomes were measured in terms of percent closure on videonasoendoscopy, percent closure on lateral VFS, and linear and angular anatomic measurements obtained from lateral VFS. Speech outcomes were measured in terms of hypernasal resonance, nasal emission, and facial grimace. There was a strong correlation between hypernasal resonance and percent closure. Further, hypernasality and facial grimaces were found to be strong indicators for large gap sizes. A moderate correlation between VFS and NE was found on the estimation of the VP gap size. They found that specific anatomic characteristics of velum were found to contribute to primary speech and velar outcome. The study concluded that instrumental assessment should be used in conjunction with perceptual speech assessment.

Scarmagnani et $\mathrm{a}^{19}$ investigated the correlation between objective pressure flow studies and perceptual speech parameters. One hundred subjects with RCLP in the age range of 6 to 47 years were considered for the study. The VP orifice area was determined using a pressure-flow technique. Hypernasality and nasal air emission were found to be significantly contributing to the VP area. These findings indicate that the VP area can provide significant information while measuring the speech outcome following cleft palate repair. There are various other factors which need to be considered while analyzing the speech characteristics in individuals with CLP. An often debated factor is the timing of palatal surgery. ${ }^{20,21}$

The timing of palatal surgery is considered as a major factor contributing to optimum outcome. The primary aim in the timing of palatal surgery is to help optimum speech output without affecting the maxillary growth. But there are wide controversies in literature regarding the same. Early age, considered in various studies, ranges from 6 months to 2 years and 2 to 6 years is generally considered as late. ${ }^{22,23}$ Hardin-Jones and Jones ${ }^{24}$ in their attempt to study speech outcome following early primary palatoplasty found that early surgery is required for the development of adequate VP function and normal speech. Few studies have also included direct observation along with perceptual speech assessment in children with early cleft palate repair. Hortis-Dzierzbicka et $\mathrm{al}^{25}$ evaluated speech outcome following a one-stage palatoplasty done at a mean age of 8.8 months. Speech samples of 28 children were collected and analyzed in terms of perceptual evaluation of hypernasality, audible nasal air emission, compensatory articulation, facial grimacing, and clinical intraoral evaluation by two experienced examiners along with NE where indicated. The analysis of speech parameters shows satisfactory results with the development of articulation, the incidence of compensatory errors, and VP function. The study concludes that well-executed early palatal repair can result in good speech outcome. Rohrich and Gosman ${ }^{26}$ in their critical review of the literature concluded that early palate closure at 10.8 months of age has an advantage of good speech outcome while late repair at around 48.6 months had a negative effect on speech outcome with no benefit on maxillary growth. Oyama et $\mathrm{a}^{27}$ found that changes in articulation can occur even after completion of speech therapy at 4 to 5 years. The study indicates that there is a need to carefully follow up these children, till 10 years or more, after primary palatoplasty.

Speech outcome has been measured successfully by various groups using different standardized protocols (e.g., Eurocleft, Scancleft, Americleft). Sell et $\mathrm{al}^{28}$ in their Clinical Standard Advisory Group (CSAG) study established that $18 \%$ of 5 -year olds had hypernasality of various degrees. The Cleft Care UK study ${ }^{29}$ established that $45 \%$ of children had nasal air emission while $10.2 \%$ had hypernasality. They concluded that speech outcome is the result of a multidisciplinary team work and not of a specialty alone.

There are very few studies which address the anatomical variation of VP structure and function in young children with RCLP. Visualizing the function of VP port and correlating it with the perceptual speech findings will provide valuable information about the efficacy of the surgical techniques and plans for further management for optimum speech.

The present study is part of an ongoing research to evaluate the speech and language outcomes in school-going children following early primary cleft palate repair. The study aimed to investigate the velar function in children with RCLP and correlate it with speech parameters. The objectives were to (1) investigate the VP function in children with RCLP using VFS images by calculating the resting gap, velar excursion, and closure ratio, (2) analyze the resonance characteristics (hypernasality and nasal air emission) using perceptual severity rating scale, and (3) find the correlation of the closure ratio with hypernasality.

\section{Method}

Twenty-five nonsyndromic, native Kannada-speaking children in the age range of 5 to 7 years ( 16 males, 9 females) who had undergone repair of the cleft palate before 18 months of age 
were selected for the study. Children who had undergone primary palatoplasty during the period 2011 to 2013 at Bhagwaan Mahaveer Jain Hospital were selected from the database and recalled for assessment during 2017 to 2018. All the children were screened for hearing, cognitive, and neurological issues. Children who had undergone two-flap palatoplasty, operated by either of two surgeons, were selected for the study. Children with cleft lip only, who had undergone primary palatal surgery later than 18 months of age, children with history of recurrent middle-ear infection, failed primary surgical results, persisting fistulas/nasal pathologies like allergic rhinitis or related conditions at the time of assessment, with evidence of severe dyspraxia/ dysarthria identified through clinical motor speech evaluation, those who did not cooperate for videofluoroscopic evaluation/ speech assessment children who attended speech therapy for more than 15 days at a stretch were excluded from the study. All children were subjected to lateral-view VFS and detailed speech assessment. Informed consent of parents was obtained prior to testing. The study was approved by the AIISH Ethics Committee.

Speech assessment was performed by a single clinician in a sound-treated room and audiovisual recording was done using a Sony digital video camera with a collar microphone. The children were seated comfortably and microphone placed approximately $10 \mathrm{~cm}$ away from the child's mouth. Children were made to repeat syllables with all pressure consonants and eight oral sentences developed by Jayakumar and Pushpavathi. ${ }^{30}$ If the child was not able to repeat the whole sentence, one more attempt was given and the best recording was considered. The recorded stimuli were subjected to perceptual assessment by using the standardized scale. The author who was part of the cleft team performed the audio-visual recording and served as the examiner as she has ample clinical experience in dealing with perceptual speech analysis. The speech samples were blinded and played in a system with headphones attached. The samples were rated by the examiner for hypernasality and nasal air emission on a 4-point severity rating scale (-Table 1) and a 2-point rating scale (-Table 2), respectively. The rating scales provided by "Universal parameters for reporting speech outcome in individuals with cleft palate"7 were used for reporting the parameters assessed in the study. The interrater reliability was established statistically. In total, $25 \%$ of the blinded speech samples were randomly selected and provided to two other experienced speech language pathologists (SLPS) trained for assessing cleft speech. The SLPs rated the samples for selected parameters of speech.
Lateral-view VFS: The function of the velum was assessed through lateral-view VFS. The lateral view represents a midsagittal plane through the VP portal that demonstrates the relationship between the velum and posterior pharyngeal wall. Speech stimuli selected for analysis were prolongation of vowel /a/ and repetition of consonant $/ \mathrm{p} /$ in the context of vowel /i/. The children were made to lie down in supine position between the fluoroscope and the image intensifier and repeat the speech stimulus when the fluoroscopy was performed by a radiologist and a speech pathologist without the use of intranasal or intraoral contrast material. The measurement was made by the author and a radiologist from the videos obtained through an inbuilt software. The ramies of the two mandibles were superimposed. The images were kept constant and were not magnified. A Sony digital recorder with a microphone was placed $30 \mathrm{~cm}$ away from the child's mouth for simultaneous audio recording of speech. The children were made to repeat the speech stimulus after the clinician while recording the videofluoroscopic images. Further, the videofluoroscopic images and the audio recordings were superimposed using Windows live movie maker. For each sample, digital screenshots during rest and maximum velar movement during the production of oral stimuli were captured from lateral videofluoroscopy (LVF). The average of the closure ratios obtained for two speech stimuli used was considered.

Linear measurements as per the guidelines proposed by an international working group ${ }^{14}$ were adhered to for measuring the gap sizes in the videofluoroscopic images. The measurements made were resting gap, velar gap, velar excursion, and closure ratio, ${ }^{14}$ and a technique of measurement was adapted from the measurement method described by Birch et al. ${ }^{15,31}$ Points were marked as shown in - Fig. 1. ${ }^{32}$

The resting gap ( - Fig. 1) was measured by drawing a line from a point on the velum when velum was at rest during quiet breathing to the point on the posterior pharyngeal wall (BD). Velar gap is the measurement made from the posterior point of the velum to the posterior pharyngeal wall when

Table 2 Severity of nasal air emission

\begin{tabular}{|l|l|}
\hline \multicolumn{2}{|l|}{ Nasal air emission } \\
\hline 0 & Audible nasal air emission absent \\
\hline 1 & $\begin{array}{l}\text { Audible nasal air emission present frequently } \\
\text { or inconsistently }\end{array}$ \\
\hline
\end{tabular}

Table 1 Severity rating for hypernasality

\begin{tabular}{|c|c|}
\hline Severity rating & Description \\
\hline 0 & $\begin{array}{l}\text { - Within normal limits } \\
\text { - Nasality does not exceed regional speech nasality and there is no perceptual evidence of cleft type } \\
\text { speech }\end{array}$ \\
\hline 1 & $\begin{array}{l}\text { - Mild } \\
\text { - Nasality exceeds regional speech nasality }\end{array}$ \\
\hline 2 & $\begin{array}{l}\text { - Moderate } \\
\text { - Hypernasality is perceived as pervasive and draws attention to itself and away from the message }\end{array}$ \\
\hline 3 & $\begin{array}{l}\text { - Severe } \\
\text { - Hypernasality is perceived as pervasive and interferes with speech understandability }\end{array}$ \\
\hline
\end{tabular}




\section{Analysis of Velar Function}

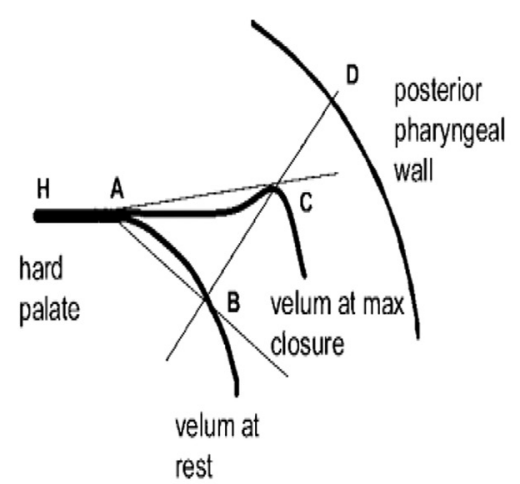

Fig. 1 Analysis of lateral view videofluoroscopic images. (Adapted from Sommerlad et al. ${ }^{32}$ )

Table 3 Scale for predicting severity of VPD using closure ratio $^{10}$

\begin{tabular}{|l|l|}
\hline Closure ratio & Severity of VP gap \\
\hline 1 & No gap, complete closure \\
\hline $0.8-0.9$ & Small gap, efficient closure \\
\hline $0.4-0.7$ & Average gap, intermediate closure \\
\hline $0.1-0.3$ & Large gap, inefficient closure \\
\hline 0 & Very large gap, lack of closure \\
\hline
\end{tabular}

Abbreviation: VPD, velopharyngeal dysfunction.

the velum is elevated to the maximum during the production of sounds (CD). The velar excursion was measured as the distance traveled by velar knee from resting position to point of maximum closure during speech. In this study, it was estimated by finding the difference between resting gap and velar gap (BD CD). The closure ratio gives the degree of velar closure as seen on lateral-view VFS, obtained as a ratio of velar excursion to resting gap, with 0 representing the velar position at rest and 1 its position at full closure contacting the posterior pharyngeal wall.

The severity of VPD is interpreted using the scale adapted by Paniagua et $\mathrm{al}^{10}$ based on the work by Lam et al ${ }^{33}$ and Golding-Kushner et al. ${ }^{14}$ The interpretations given in - Table 3 were based on closure quality and gap size obtained in VFS. In the present study, the closure ratio was interpreted using the same scale. The closure ratio was further correlated with severity of hypernasality and nasal air emission.

Interrater reliability was established for $25 \%$ of the cases statistically. Two other SLPs experienced in videofluoroscopic assessment for VP function in CLP were selected. The extracted images were randomized and provided to the SLPs to calculate resting and velar gaps. Instructions were given on the method of measurements. Reliability data were not established for velar excursion and closure ratio as these are dependent on other two measurements.

\section{Results}

Velopharyngeal Function in Children with RCLP using LVF

Reliability of the measurements made from videofluoroscopic images was established by comparing the measurements made by the primary investigator with those of other two SLPs for resting and velar gaps. Interclass correlation coefficient (ICC) was calculated. A high degree of reliability was found between three raters for both the measurements. The average measure ICC was 0.97 with $95 \%$ confidence interval from 0.88 to 0.99 for the resting gap. The average measure ICC was 0.80 with a $95 \%$ confidence interval from 0 to 0.97 for the velar gap.

The parameters extracted from the LVF provide details of the velar gap present at rest, the extent of velar movement, and closure ratio. Median values and interquartile range for all the parameters are given in - Table 4 .

The raw data compared with the observations on VFS images showed enlarged adenoid pads in two children with a minimum resting gap $(2 \mathrm{~mm})$. Six children with resting gap of 2 to $2.5 \mathrm{~mm}$ did not exhibit a velar gap. Two children with a $3.5-\mathrm{mm}$ resting gap had a $2-\mathrm{mm}$ velar gap. There was no such relationship observed in the rest of the children.

The data obtained on various parameters were classified based on the severity classification proposed by Lam et al. ${ }^{33}$ The raw data showed that $52 \%$ of the children in the group had adequate VP closure (out of this $48 \%$ had complete VP closure and $4 \%$ had a small gap but efficient closure). The closure ratio measured from the videofluoroscopic images for this group ranged from 0.8 to 1 . Out of the remaining $48 \%, 40 \%$ of children had an average VP gap and intermediate VP closure (closure ratio: $0.4-0.7$ ), while $8 \%$ of children had a large gap and inefficient closure (closure ratio: 0.1-0.3; - Fig. 2).

\section{Resonance Characteristics}

Reliability of the assessment of speech samples was established by conducting an interrater reliability in $25 \%$ of the sample. Kendall's $W$ test was done to establish interrater reliability between the three examiners. There was a significantly high agreement between the raters for hypernasality ((Kendall's $W=0.963, p=0.02$ ) and nasal air emission (Kendall's $W=1, p=0.017$ ).

The resonance parameter hypernasality was assessed using a 4-point rating scale and nasal air emission using a 2-point rating scale. The average hypernasality score was 1.13 on a 4 -point rating scale. Majority (52\%) of the

Table 4 Median and IQR for VFS parameters

\begin{tabular}{|l|l|l|}
\hline Parameter & Median & IQR \\
\hline Resting gap & $4 \mathrm{~mm}$ & $3.5-5 \mathrm{~mm}$ \\
\hline Velar gap & $1 \mathrm{~mm}$ & $0-3 \mathrm{~mm}$ \\
\hline Velar excursion & $2 \mathrm{~mm}$ & $2-6 \mathrm{~mm}$ \\
\hline Closure ratio & 0.8 & $0.46-1$ \\
\hline
\end{tabular}

Abbreviations: IQR, interquartile range; VFS, videofluoroscopy. 


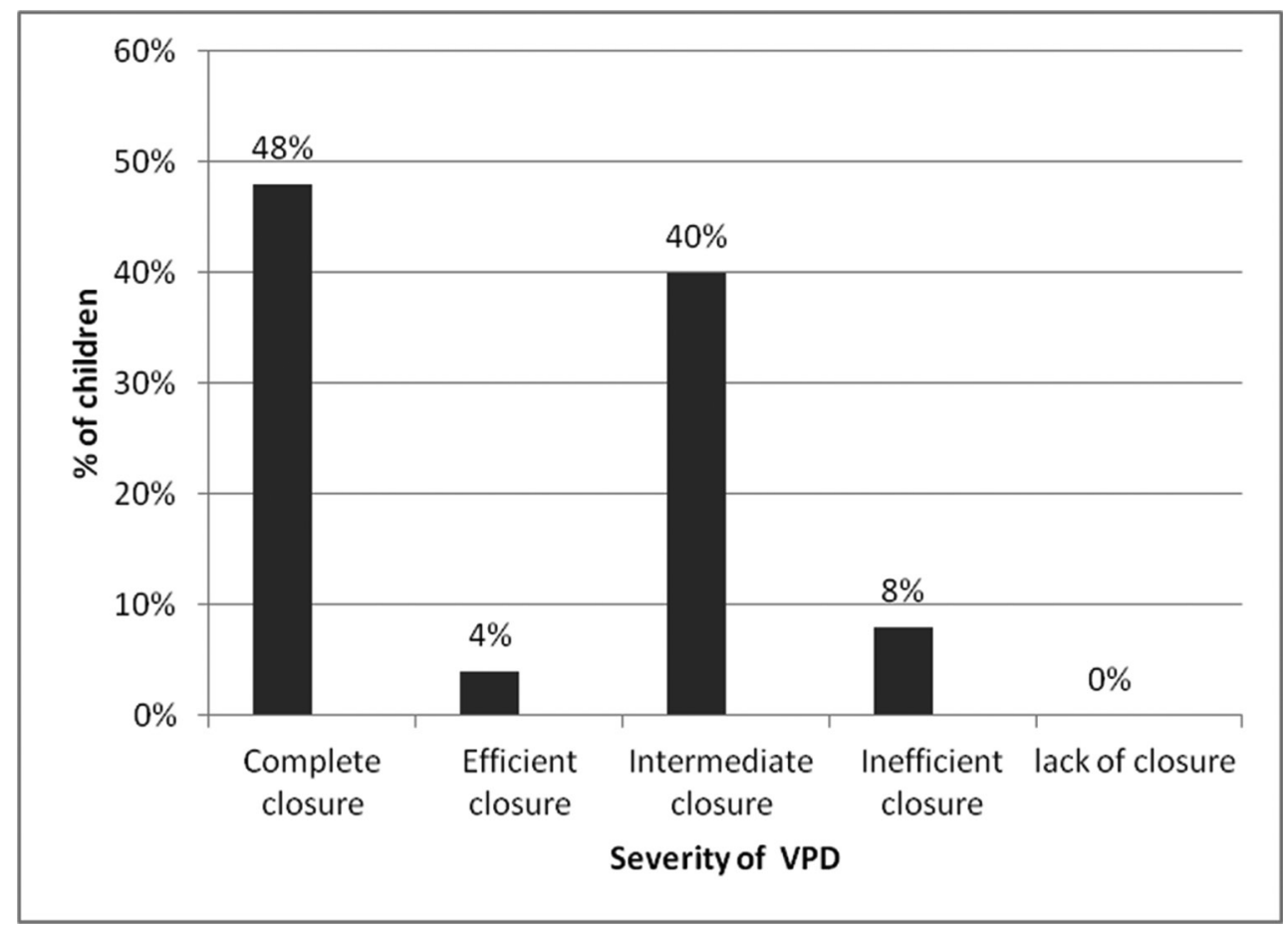

Fig. 2 Severity of velopharyngeal dysfunction.

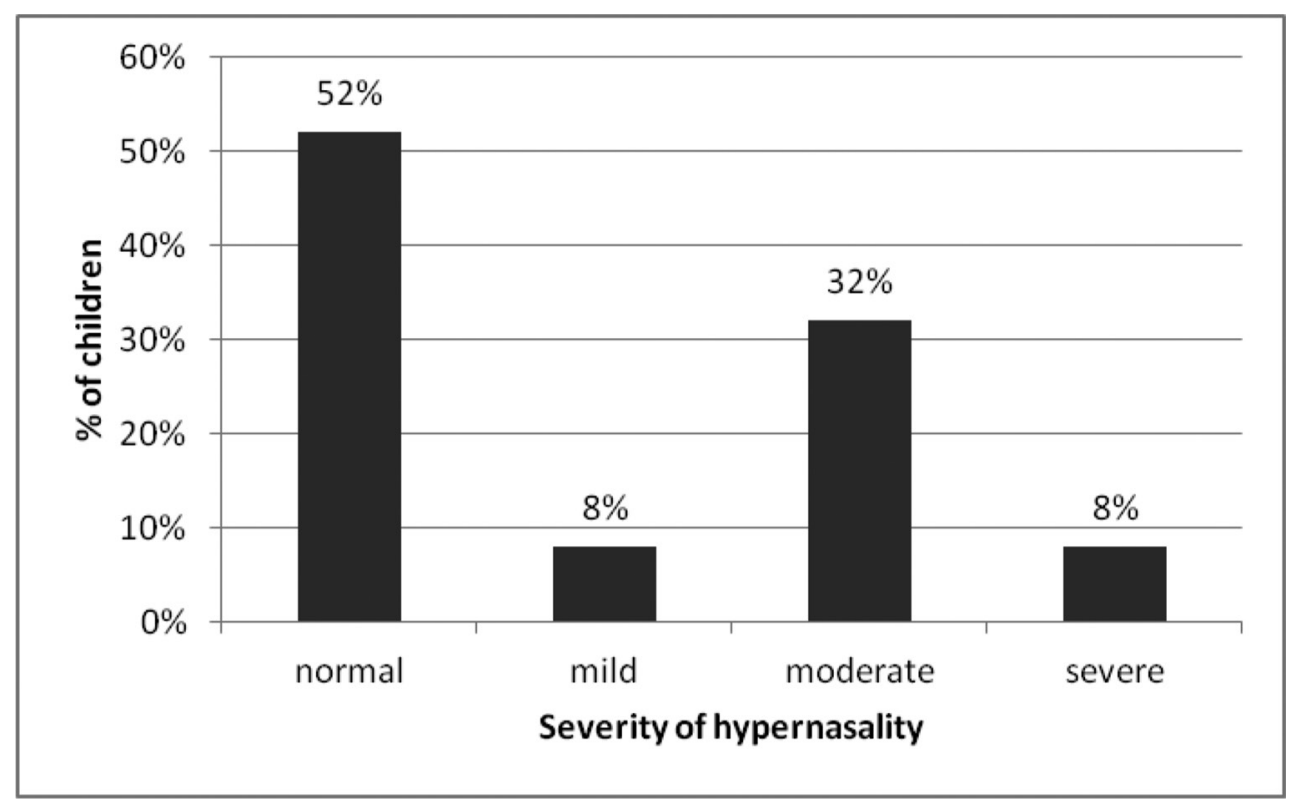

Fig. 3 Severity of hypernasality.

children exhibited normal resonance, $32 \%$ had moderate hypernasality, and $8 \%$ had mild hypernasality, while $8 \%$ were rated to have severe hypernasality ( - Fig. 3 ).

Forty-four per cent (11) of children exhibited nasal air emission while in $56 \%$ (14) it was absent (-Fig. 4).

\section{Correlation of Closure Ratio and Resonance Parameters}

The first objective of the study which was done by extracting the parameters from VFS reflected the structural competency. The second objective reflected the functional aspects of the structures assessed. To find the correlation between the structural competency and functional attributes, correlations between the closure ratio and hypernasality and nasal air emission were studied. Spearman's correlation was run to assess the relationship between the closure ratio and hypernasality. The results showed a strong $(p<0.001)$ negative correlation between the closure ratio and hypernasality $\left(r_{s}=0.846, p=0.000\right)$. As the closure ratio increases, hypernasality reduces. The correlations of various parameters assessed in the study are given in - Table 5. This table shows that the correlation of severity of VPD with hypernasality is good for normal and 


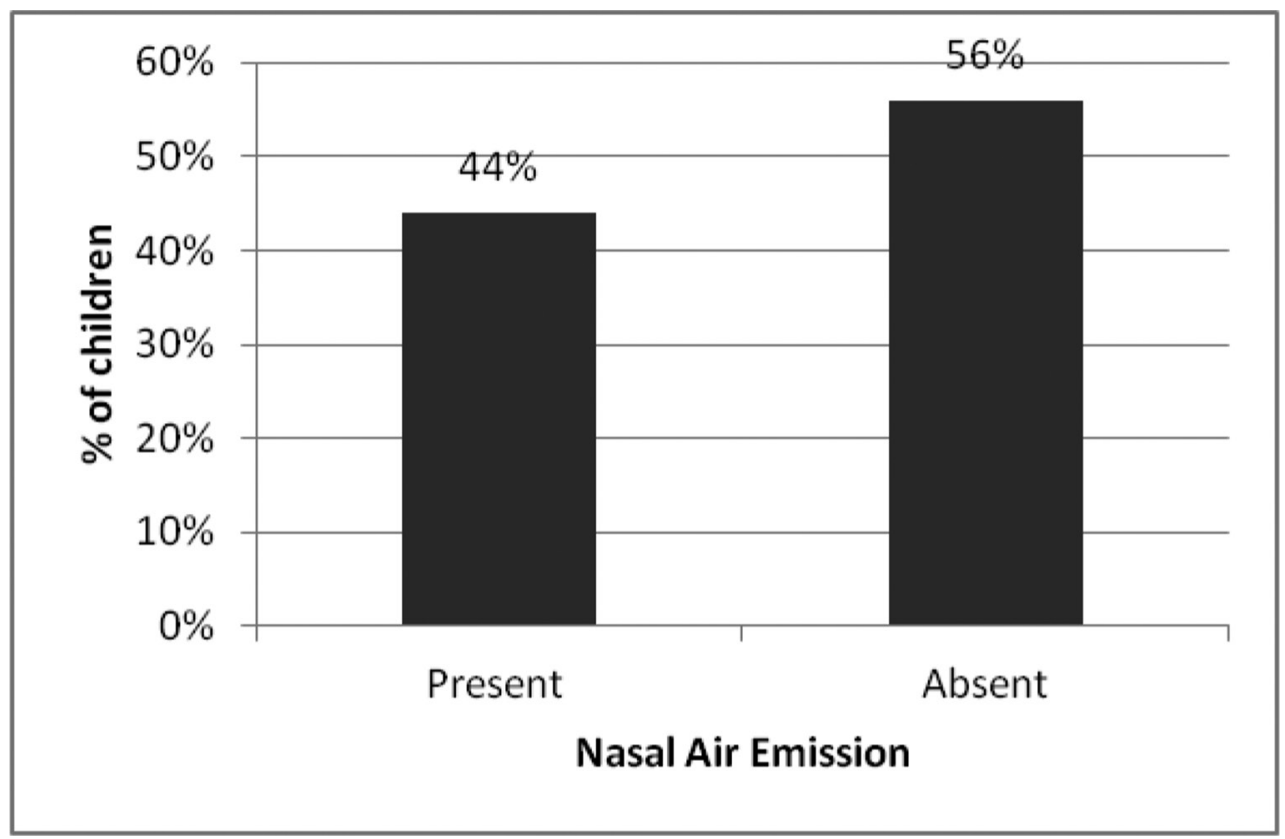

Fig. 4 Rating for nasal air emission.

Table 5 Structural and functional correlation of velopharyngeal mechanism

\begin{tabular}{|l|l|l|l|}
\hline \multicolumn{2}{|c|}{ Structural correlate of velopharyngeal function } & \multicolumn{2}{c|}{ Functional attributes of velopharyngeal function } \\
\hline Closure based on closure ratio & $\%$ of children & Hypernasality & Nasal air emission (NAE) \\
\hline No gap, complete closure & $48 \%$ & $\begin{array}{l}\text { Normal 40\% } \\
\text { Mild 8\% }\end{array}$ & No cases with NAE \\
\hline Small gap, efficient closure & $4 \%$ & Normal 4\% & No cases with NAE \\
\hline Average gap, intermediate closure & $40 \%$ & $\begin{array}{l}\text { Normal 4\% } \\
\text { Moderate 32\% } \\
\text { Severe 4\% }\end{array}$ & $\begin{array}{l}36 \% \text { present } \\
4 \% \text { absent }\end{array}$ \\
\hline Large gap, inefficient closure & $8 \%$ & $\begin{array}{l}\text { Moderate 4\% } \\
\text { Severe 4\% }\end{array}$ & $8 \%$ present \\
\hline
\end{tabular}

severe categories. Mild structural deviation may not have a significant effect on the resonance. The raw data show that nasal air emission is not present in $48 \%$ of children with no gap, complete closure, and normal nasality.

\section{Discussion}

The study involved 25 children with RCLP who underwent early surgery. Structural aspects of VP function were assessed using LVF and the functional attributes using a rating scale. The correlations of the structural and functional aspects were also assessed. Subjective analysis of the VFS images showed that majority of the children had no gap-complete closure followed by average gap-intermediate closure, large gap- inefficient closure, and small gap-efficient closure. The results showed that there were very few children with a large gap size. This indicates the efficacy of primary palatoplasty and predicts the success of further secondary corrections if required. The VFS findings in the present study show an average resting gap of $4.08 \mathrm{~mm}$. Yellinedi and Damalacheru$\mathrm{vu}^{34}$ reported that there are high chances of having maximal velar closure for speech when resting gap is $<6 \mathrm{~mm}$. None of the children in the present study group had an immobile velum. Both these findings show successful repair of levator veli palatini muscle during primary palatoplasty. The present study supports the findings of previous studies ${ }^{24-26}$ that early surgery will result in better speech outcome in terms of competent VP function.

Another interesting point that was noted here was the presence of enlarged adenoid pads in two children with a minimum resting gap. Literature indicates that the size of the adenoids reduces as the age increases and it disappears by the age of 8 to 9 years. Various studies ${ }^{35,36}$ show that the adenoid provides support for VP closure in young children. Hence it is called velum-adenoid closure. Similar findings were observed in a few children in the present study, which warrants further follow-up. The findings also support previous studies contradicting adenoidectomy in children with RCLP as it may result in VPD. ${ }^{37}$

The second part of the observation was the functional attributes of the VP closure mechanism. The results show that out of 25 children majority exhibited normal nasality followed by moderate hypernasality, severe hypernasality, and mild nasality. Forty-four percent of children 
having nasal air emission in the study group were comparable with the findings of a large-scale study by Sell ${ }^{4}$ (where $45 \%$ of children had nasal air emission) and lesser than the small sample studied by Hubbard et $\mathrm{al}^{9}$ (66\% of children had nasal air emission). This also indicates that early surgery has an impact on reducing the incidence of abnormal resonance. ${ }^{24-26}$ However, there are some studies contradicting this claim indicating that the timing of surgery does not play any role. ${ }^{38}$

The third finding observed in the study was the significant correlation obtained between the structural and functional aspects of the VP mechanism. Significant correlation was found wherein subjects who exhibited a normal closure also showed normal nasality and absence of nasal air emission. The result indicates that the closure ratio and resonance parameters are well correlated and as the velar closure increases nasality and nasal emission reduces. The findings are in consensus with the study by Lipira et al $^{11}$ and Scarmagnani et $\mathrm{al}^{19}$ who showed that hypernasality and nasal air emissions are strong predictors of the VP gap size. Some subjects who exhibited a mild gap in LVF also exhibited hypernasality while the correlation was complete in the severe category. This is in agreement with the findings of other studies that show that hypernasality best predicts small and large gap sizes. ${ }^{17,19}$

The present study is an initial attempt to find out the impact of early surgery on various speech parameters and their structural correlates. The results show the effectiveness of employing instrumental evaluation in conjunction with perceptual speech assessment in early age, for timing appropriate management. The results need to be evaluated in a larger population and compared with data from other centers.

\section{Summary and Conclusion}

Speech outcome following different procedures and timing of cleft palate surgery is warranted for every center to improve the quality and to make clinical audits possible. The study provides insights into the method for effectively utilizing the VFS images to obtain anatomical information. This helps in understanding the degree of structural deficit persisting, which helps the surgeon refine on the techniques. Establishing a correlation between perceptual assessment and instrumental assessment will help in refining the reliability and subjectivity of perceptual assessment. The data on a larger population and more speech parameters will help in identifying the percentage of children with normal speech and also help in deciding the best practice guidelines.

\section{Financial Support and Sponsorship}

Nil.

\section{Conflict of Interest}

There are no conflicts of interest.

\section{References}

1 Bressmann T, Sader R. Nasality and nasalence. Logopedia 2000;8:22-33
2 McWilliams BJ, Morris HL, Shelton RL, Cleft Palate Speech. Philadelphia, PA: B.C. Decker;1990

3 Lohmander A, Olsson M. Methodology for perceptual assessment of speech in patients with cleft palate: a critical review of the literature. Cleft Palate Craniofac J 2004;41(1):64-70

4 Sell D. Issues in perceptual speech analysis in cleft palate and related disorders: a review. Int J Lang Commun Disord 2005;40(2):103-121

5 Sell D, John A, Harding-Bell A, Sweeney T, Hegarty F, Freeman J. Cleft audit protocol for speech (CAPS-A): a comprehensive training package for speech analysis. Int J Lang Commun Disord 2009;44(4):529-548

6 Howard S, Heselwood B. Instrumental and perceptual phonetic analyses: the case for two-tier transcriptions. Clin Linguist Phon 2011;25(11-12):940-948

7 Henningsson G, Kuehn DP, Sell D, Sweeney T, Trost-Cardamone JE, Whitehill TL; Speech Parameters Group. Universal parameters for reporting speech outcomes in individuals with cleft palate. Cleft Palate Craniofac J 2008;45(1):1-17

8 Shprintzen RJ, The velopharyngeal mechanism. In: Berkowitz S, ed. Cleft Lip and Palate: Diagnosis and Management. 2nd ed. Berlin: Springer; 2005:643-656

9 Hubbard BA, Rice G, Muzaffar AR. Contractility of sphincter pharyngoplasty: relevance to speech outcomes. Can J Plast Surg 2013;21(1):15-18

10 Paniagua ML, Collares MV, da Costa SS. Comparative study of three techniques of palatoplasty in patients with cleft of lip and palate via instrumental and auditory-perceptive evaluations. Int Arch Otorhinolaryngol 2010;14:18-31

11 Lipira AB, Grames LM, Molter D, Govier D, Kane AA, Woo AS. Videofluoroscopic and nasendoscopic correlates of speech in velopharyngeal dysfunction. Cleft Palate Craniofac J 2011;48(5):550-560

12 Poppelreuter S, Engelke W, Bruns T. Quantitative analysis of the velopharyngeal sphincter function during speech. Cleft Palate Craniofac J 2000;37(2):157-165

13 Witt PD, Marsh JL, McFarland EG, Riski JE. The evolution of velopharyngeal imaging. Ann Plast Surg 2000;45(6):665-673

14 Golding-Kushner KJ, Argamaso RV, Cotton RT, et al. Standardization for the reporting of nasopharyngoscopy and multiview videofluoroscopy: a report from an International Working Group. Cleft Palate J 1990;27(4):337-347, discussion 347-348

15 Birch MJ, Sommerlad BC, Fenn C, Butterworth M. A study of the measurement errors associated with the analysis of velar movements assessed from lateral videofluoroscopic investigations. Cleft Palate Craniofac J 1999;36(6):499-507

16 Kummer AW, Curtis C, Wiggs M, Lee L, Strife JL. Comparison of velopharyngeal gap size in patients with hypernasality, hypernasality and nasal emission, or nasal turbulence (rustle) as the primary speech characteristic. Cleft Palate Craniofac J 1992;29(2):152-156

17 Kummer AW, Briggs M, Lee L. The relationship between the characteristics of speech and velopharyngeal gap size. Cleft Palate Craniofac J 2003;40(6):590-596

18 Smith BE, Kuehn DP. Speech evaluation of velopharyngeal dysfunction. J Craniofac Surg 2007;18(2):251-261, quiz 266-267

19 Scarmagnani RH, Barbosa DA, Fukushiro AP, Salgado MH, Trindade IEK, Yamashita RP. Relationship between velopharyngeal closure, hypernasality, nasal air emission and nasal rustle in subjects with repaired cleft palate. CoDAS 2015;27(3):267-272

20 Kirschner RE, Randall P, Wang P, et al. Cleft palate repair at 3 to 7 months of age. Plast Reconstr Surg 2000;105(6):2127-2132

21 Ha S, Koh KS, Moon H, Jung S, Oh TS. Clinical outcomes of primary palatal surgery in children with non-syndromic cleft palate with and without lip. BioMed Res Int 2015; 2015:185459 
22 Brunnegård K, Lohmander A. A cross-sectional study of speech in 10-year-old children with cleft palate: results and issues of rater reliability. Cleft Palate Craniofac J 2007;44(1):33-44

23 Murthy J, Sendhilnathan S, Hussain SA. Speech outcome following late primary palate repair. Cleft Palate Craniofac J 2010;47(2):156-161

24 Hardin-Jones MA, Jones DL. Speech production of preschoolers with cleft palate. Cleft Palate Craniofac J 2005;42(1):7-13

25 Hortis-Dzierzbicka M, Radkowska E, Fudalej PS. Speech outcomes in 10-year-old children with complete unilateral cleft lip and palate after one-stage lip and palate repair in the first year of life. J Plast Reconstr Aesthet Surg 2012;65 (2):175-181

26 Rohrich RJ, Gosman AA. An update on the timing of hard palate closure: a critical long-term analysis. Plast Reconstr Surg 2004;113(1):350-352

27 Oyama T, Sunakawa H, Arakaki K, et al. Articulation disorders associated with maxillary growth after attainment of normal articulation after primary palatoplasty for cleft palate. Ann Plast Surg 2002;48(2):138-147

28 Sell D, Grunwell P, Mildinhall S, et al. Cleft lip and palate care in the United Kingdom-the Clinical Standards Advisory Group (CSAG) Study. Part 3: speech outcomes. Cleft Palate Craniofac J 2001;38(1):30-37

29 Sell D, Mildinhall S, Albery L, Wills AK, Sandy JR, Ness AR. The cleft care UK study. Part 4: perceptual speech outcomes. Orthod Craniofac Res 2015;18(Suppl 2):36-46

30 Jayakumar T, Pushpavathi M, Normative Score for Nasometer in Kannada. Student Research at AIISH (Articles Based on Dissertation Done at AIISH) 2015;VII: 44-53
31 Birch M, Sommerlad BC, Bhatt A. Image analysis of lateral velopharyngeal closure in repaired cleft palates and normal palates. Br J Plast Surg 1994;47(6): 400-405

32 Sommerlad BC, Fenn C, Harland K, et al. Submucous cleft palate: a grading system and review of 40 consecutive submucous cleft palate repairs. Cleft Palate Craniofac J 2004;41(2):114-123

33 Lam E, Hundert S, Wilkes GH. Lateral pharyngeal wall and velar movement and tailoring velopharyngeal surgery: determinants of velopharyngeal incompetence resolution in patients with cleft palate. Plast Reconstr Surg 2007; 120(2):495-505

34 Yellinedi R, Damalacheruvu MR. Is there an optimal resting velopharyngeal gap in operated cleft palate patients? Indian J Plast Surg 2013;46(1):87-91

35 Calnan JS. Movements of the soft palate. Br J Plast Surg 1953;5(4):286-296

36 Subtelny JD, Baker HK. The significance of adenoid tissue in velopharyngeal function. Plast Reconstr Surg (1946) 1956;17(3):235-250

37 Hubbard BA, Rice GB, Muzaffar AR. Adenoid involvement in velopharyngeal closure in children with cleft palate. Can J Plast Surg 2010;18(4):135-138

38 Lohmander-Agerskov A, Söderpalm E, Friede H, Lilja J. A longitudinal study of speech in 15 children with cleft lip and palate treated by late repair of the hard palate. Scand J Plast Reconstr Surg Hand Surg 1995;29(1):21-31 